\title{
An audit on completeness of reporting Whipple's specimens
}

\author{
Pelzom T ${ }^{1}$, Tillakaratne $\mathrm{MSB}^{2}$, Jaleel MSA ${ }^{1}$, Pathirana $\mathrm{A}^{3}$, Siriwardana $\mathrm{RC}^{2}$ \\ ${ }^{1}$ Department of Surgery, Colombo North Teaching Hospital, Ragama, Sri Lanka \\ ${ }^{2}$ Department of Surgery, Faculty of Medicine, University of Kelaniya, Ragama, Sri Lanka \\ ${ }^{3}$ Department of Surgery, Faculty of Medical Sciences, University of Sri Jayewardenepura, Sri Lanka
}

Keywords: Whipple's; histopathology; synoptic reporting; pancreatic cancer; survival

\begin{abstract}
\section{Introduction}

This audit is focused on assessing the completeness of vital information in traditional reports of Whipple's procedure, using the Royal College of Pathologists data sets for pancreatic cancer reporting as the benchmark. We believe a standardized reporting system will take into account significant variables that may impact treatment quality.
\end{abstract}

\section{Methodology}

This is a descriptive cross sectional study. A hundred and forty-three Whipple's histopathological reports were examined and compared to The Royal College of Pathologists data set for reporting of carcinomas of pancreas, ampulla of Vater and common bile duct.

\section{Results}

The length of the reports varied markedly with the shortest report having 156 words and the longest report having 1095 words. The median word count was 385 words. The frequency of reporting the variables varied too. Type of tumour was documented in $100 \%$ of reports whereas variables such as nodal stage and superior mesenteric artery resection margin were reported in only $76.9 \%$ and $35 \%$ of reports respectively, both having direct implications on prognosis. Further the frequency of reporting of the background pathology was low as $24.5 \%$.

\section{Conclusions}

Due to the descriptive nature of the traditional pathological reporting system, some of the significant variables can be missed while converting what is observed in to a report. This may impact adversely in planning adjuvant treatment and evaluation of prognosis after surgery. Adherence to a standardized synoptic reporting system may help to overcome this drawback.

\footnotetext{
Correspondence: Rohan Chaminda Siriwardana

E-mail: rohansiriwardana@yahoo.com

Received: 07-02-2020Accepted: 26-04-2020

(i) http://orcid.org/0000-0002-6477-2072

DOI: http://doi.org/10.4038/sljs.v38i1.8687
}

The Sri Lanka Journal of Surgery 2020; 38(1): 18-21

\section{Introduction}

Pancreatic cancer is one of the leading causes for cancerrelated deaths worldwide [1].

Comprehensive and accurate reporting of pathological specimens in pancreatic cancer is important in confirming the diagnosis and predicting the prognosis of the patients. This in turn helps in planning adjuvant therapy and follow up of the patient. It also helps in evaluating quality of services such as surgery and radiology. Accurate pathology reporting also contributes to the development of adjuvant therapy and facilitates high quality research $[2,3]$.

Whipple specimen is a complex sample that includes multiple margins. The establishment of a general consensus of nomenclature, definitions and standardized protocol of pathological reporting for Whipple specimen is crucial especially considering the complexity and the extent of different variables in the specimen.

In Sri Lanka reporting of Whipple specimen is still performed using traditional descriptive reports. This audit assessed the completeness of vital information in traditional reports against The Royal College of Pathologists data set for pancreatic cancer reporting [4].

\section{Methodology}

This is a descriptive cross sectional study. All histopathology reports of Whipple surgery performed from 2011 to 2019 for at Colombo North Teaching Hospital [CNTH] and Colombo South Teaching Hospital [CSTH] were reviewed and reports with malignant disease were selected. There were total of 143 reports. In both centres, standard pylorus resecting Whipple surgery was performed. Uncinated process was completely resected from the superior mesenteric artery and the margins marked. All had standard lymphadenectomy [stations 5, 6, 8a, 12b1, 12b2, 12c, 13a, 13b, 14a, 14b, 17a, and 17b.] [5, 6].

The specimens were fixed in formaldehyde and examined. After identification and measurements of gross anatomical structures, the tumour was identified and the tumour site, size, type, grading, its relationship to the surrounding structures and the transection margins were recorded. Lymph nodes were also sampled. 
The Royal College of Pathologists data set for reporting of carcinomas of pancreas, ampulla of Vater and common bile duct, which constitute of macroscopic and microscopic core data sets were used as a guide for reporting. Macroscopic core data set includes six items type of specimen, site of tumour, maximum tumour dimension, resection margins, and presence of a named vessel and background pathology. Microscopic core data includes histological type of tumour, histological differentiation, size and maximum extent of local invasion, peri neural invasion, named vessel involvement, lymph node status, resection margin status, regression following neo adjuvant therapy, background abnormalities, completeness of resection, TNM stage and SONMED CT [Systematized Nomenclature of Medicine Clinical Terms] codes.[7] However, standard unstructured report was produced as the final product after evaluating slides.

All 143 pathology reports were studied by an MBBS qualified doctor. Twenty-two of variables that are in accordance with The Royal College of Pathologists guidelines were looked for in each of the reports using a checklist. Complete pathology report was read minimum of two times and picked up points were highlighted. Subsequently each variable was entered in to a separate SPSS [for WindowsTM Version 16.0, SPSS, Inc., Chicago, IL, USA] database. Each variable was then presented as frequencies indicating whether they reported or unreported and analysed as percentages. The word count of each report was recorded and the median was calculated.

\section{Results}

The median age of the group was 56 years [range 17 - 81] and $72 \%$ were males. The length of the reports varied markedly with the shortest report having 156 words and the longest report having 1095 words. The median word count was 385 words.

Type of tumour was documented in all the reports [100\%] and the site of the tumour was reported in $93.3 \%$ of the time. Pancreatic and bile duct transection margins were reported in $94.4 \%$ and $89.5 \%$ of the reports respectively. Posterior dissection margin was reported in $76.2 \%$ and the anterior dissection margin was reported in $71.3 \%$ only. The reporting on the SMA dissection margin was even less with only $35 \%$ of the reports having the data. Size of the tumour was documented in $90.9 \%$ of the specimens. Tumour type was documented in $100 \%$ of the reports. However, the background pathology was only recorded in $24.5 \%$ of the specimens. Lymphovascular invasion and perineural invasion was reported in $79.7 \%$ and $79 \%$ respectively.

Total number of lymph nodes harvested was documented only in $89.5 \%$ of the patients. Number of involved lymph nodes was documented in $99.3 \%$. However, the $\mathrm{N}$ stage was documented only in $76.9 \%$ of the reports. Tumour differentiation was documented only in $62.9 \%$ of the reports.

\section{Discussion}

The Whipple's specimen is unique and complex sample due to the three dimensional arrangement of the adjacent structures and margins [8]. It has multiple resection margins. Transection margins are those of pancreatic neck, common bile duct, superior mesenteric artery, jejunum and stomach.

Table 1. The number of reported and non-reported variables were tabulated

\begin{tabular}{|l|l|}
\hline Variable & $\begin{array}{l}\text { Reported percentage } \\
\text { (N=143) }\end{array}$ \\
\hline 1.Site of the tumor & 93.7 \\
\hline 2.Type of the specimen & 100 \\
\hline 3.Maximum tumor diameter & 90.9 \\
\hline 4.Macroscopic margin involvement & 81.8 \\
\hline 5.Histologic Type & 100 \\
\hline 6.Tumor differentiation/grading & 62.9 \\
\hline 7.Maximum extent of invasion (T) & 98.4 \\
\hline 8.Gastric transection margin & 93 \\
\hline 9.Duodenal transection margin & 93 \\
\hline 10.Pancreatic transection margin & 94.4 \\
\hline 11.Bile duct transection margin & 89.5 \\
\hline 12.SMV, SMA dissection margin & 35 \\
\hline 13.Posterior dissection margin & 76.2 \\
\hline 14.Anterior dissection margin & 71.3 \\
\hline 15.Lymphovascular invasion & 79.7 \\
\hline 16.Perineural invasion & 79 \\
\hline 17.Total number of nodes & 76.9 \\
\hline 18.Total number of nodes involved & 99.3 \\
\hline 19.N stage & \\
\hline 20.Background pathology & \\
\hline 21.Pathological TNM staging & \\
\hline & \\
\hline & \\
\hline
\end{tabular}


The dissection margins are the superior mesenteric vein margin and the posterior margin overlying the aorto-caval groove. Anterior surface is not a true dissection margin but its involvement is known to increase the local recurrence [9]. Whipple surgery for malignant disease carries a variable prognosis influenced by many factors. Offering adjuvant treatment is an important decision after surgery [3]. For both these, resected specimen holds many answers.

$\mathrm{R}$ - 1 resection of the SMA margin is a known risk factor for poor prognosis after pancreatic cancer resection [9, 10]. Reporting of the SMA margin was low as $35 \%$ in the reports that were analysed. The reporting on other dissection margins was also less compared to the pancreatic transection margin. Margin status is an important parameter in deciding on adjuvant treatment [3]. Histological grading and the degree of differentiation have shown a clear impact on the prognosis in most studies [11]. The reporting on histological grading was $62.9 \%$, which is less compared to the percentage of reporting on other variables.

Background pancreatic pathology was reported only in $24.5 \%$ of cases that we evaluated. Knowing the background status of the pancreas is important in the follow-up after surgery especially when there is auto immune pancreatitis, atrophy and fibrosis [12]. Adenocarcinomas originating from the ampulla of Vater is known to have either intestinal differentiation or pancreatico biliary type differentiation with latter type having a poorer prognosis [13]. This information was not available in most of the reports.

Intrapancreatic perineural invasion and extra pancreatic neural plexus invasion are correlated. This is identified as a major cause for local recurrence [14] which was not uniformly documented in the reports. Lymph nodes are another important area of assessment. Though the number of nodes positive was stated total number harvested nodes were inconsistent. The rates of reporting on lympho vascular and perineural invasion were less than $80 \%$ in the sample. These parameters related to nodes are considered as important prognostic markers [15-18].

Value of adjuvant chemotherapy is well recognized in pancreatic cancer [3]. It is recommended in patients with poor prognostic tumours indicated by surrogate markers in the pathology specimen [9]. Missing valuable data in the specimen can sometime affect this important decisionmaking.

Traditional report has almost 400 words typed as a description. We observed that typing process itself takes significant time of a computer operator. By using synoptic reporting chances of missing variables we observed could be minimized and the time taken to type and read a report can be reduced.

In conclusion, due to the descriptive nature of the traditional pathological reporting system, some of the significant variables can be missed while converting what is observed in to a report. This may impact adversely in planning adjuvant treatment and evaluation of prognosis after surgery. Adherence to a standardized synoptic reporting system may help to overcome this drawback.

\section{Ethical Clearance}

The study was conducted in accordance to the Helsinki Declaration of 1975 , as revised in 2000 .

All authors disclose no conflict of interest. The study was conducted in accordance with the ethical standards of the relevant institutional or national ethics committee and the Helsinki Declaration of 1975, as revised in 2000.

\section{References}

1. Bray F, Ferlay J, Soerjomataram I, Siegel RL, Torre LA, Jemal A. Global cancer statistics 2018: GLOBOCAN estimates of incidence and mortality worldwide for 36 cancers in 185 countries. CA Cancer J Clin. 2018 Sep;68:394-424.

https://doi.org/10.3322/caac.21492

2. Gill AJ, Johns AL, Eckstein R, et al. Synoptic reporting improves histopathological assessment of pancreatic resection specimens. Pathology. 2009 Feb;41:161-167.

https://doi.org/10.1080/00313020802337329

3. Conroy T, Ducreux M. Adjuvant treatment of pancreatic cancer. Curr Opin Oncol. 2019 Jul;31(4):346-353. https://doi.org/10.1097/CCO.0000000000000546

4. Campbell F, Cairns A, Duthie F, Feakins R. Dataset for histopathological reporting of carcinomas of the pancreas, [Internet]. London: Royal College of Pathologists; 2019 [cited 2020 Feb 3]. Available from:

https:/www.rcpath.org/uploads/assets/34910231-c106-4629a2de9e9ae6f87ac1/G091-Dataset-for-histopathologicalreporting-of-carcinomas-of-the-pancreas-ampulla-of-Vater-andcommon-bile-duct.pdf

5. Farnell MB, Aranha GV, Nimura Y, Michelassi F. The role of extended lymphadenectomy for adenocarcinoma of the head of the pancreas: Strength of the evidence. J Gastrointest Surg. 2008 Apr;12:651-656. https://doi.org/10.1007/s11605-007-0451-1

6. Kawarada Y, Isaji S. Stage classifications of pancreatic cancer: comparison of the Japanese and UICC classifications and proposal for a new staging system. Union Internationale Contre le Cancer. Pancreas. 1998 Apr;16:255-264.

https://doi.org/10.1097/00006676-199804000-00009

7. Carter KJ, Rinehart S, Kessler E, et al. Quality Assurance in Anatomic Pathology: Automated SNOMED Coding. J Am Med Inform Assoc. 1996 Jul-Aug;3:270-272.

https://doi.org/10.1136/jamia.1996.96413134 
8. Campbell F, Smith RA, Whelan P, et al. Classification of R1 resections for pancreatic cancer: The prognostic relevance of tumour involvement within $1 \mathrm{~mm}$ of a resection margin. Histopathology. 2009 Sep;55:277-283.

https://doi.org/10.1111/j.1365-2559.2009.03376.x

9. Tempero MA. NCCN Guidelines Updates: Pancreatic Cancer. J Natl Compr Canc Netw. 2019 May;17:603-605. https://doi.org/10.6004/jnccn.2019.0003

10. Delpero JR, Jeune F, Bachellier P, et al. Prognostic Value of Resection Margin Involvement after Pancreaticoduodenectomy for Ductal Adenocarcinoma. Ann Surg. 2017 Nov;266:787-796. https://doi.org/10.1097/SLA.0000000000002432

11 Andrianello S, Paiella S, Allegrini V, et al. Pancreaticoduodenectomy for distal cholangiocarcinoma: Surgical results, prognostic factors, and long-term follow-up. Langenbeck's Arch Surg. 2015 Jul;400:623-628. https://doi.org/10.1007/s00423015-1320-0

12.Tanaka M, Fernández-Del Castillo C, Adsay V, et al. International consensus guidelines 2012 for the management of IPMN and MCN of the pancreas. Pancreatology. 2012 May-Jun;12:183197. https://doi.org/10.1016/j.pan.2012.04.004

13. Tran KTC, Smeenk HG, Van Eijck CHJ, et al. Pylorus preserving pancreaticoduodenectomy versus standard whipple procedure: A prospective, randomized, multicenter analysis of 170 patients with pancreatic and periampullary tumors. Ann Surg. 2004 Nov;240:738-745.

https://doi.org/10.1097/01.sla.0000143248.71964.29
14.Kayahara M, Nagakawa T, Konishi I, Ueno K, Ohta T, Miyazaki I. Clinicopathological study of pancreatic carcinoma with particular reference to the invasion of the extrapancreatic neural plexus. Int J Pancreatol. 1991 Oct; 10:105-111.

15.Basturk O, Saka B, Balci S, et al. Substaging of Lymph Node Status in Resected Pancreatic Ductal Adenocarcinoma Has Strong Prognostic Correlations: Proposal for a Revised N Classification for TNM Staging. Ann Surg Oncol.2015 Dec; 22:S1187-1195.https://doi.org/10.1245/s10434-015-4861-0

16. Contreras CM, Lin CP, Oster RA, et al. Increased pancreatic cancer survival with greater lymph node retrieval in the National Cancer Data Base. Am J Surg. 2017 Sep ;214:442-449. https://doi.org/10.1016/j.amjsurg.2017.06.036

17.Schwarz RE, Smith DD. Extent of lymph node retrieval and pancreatic cancer survival: Information from a large US population database. Ann Surg Oncol. 2006 Sep;13:1189-1200. https://doi.org/10.1245/s10434-006-9016-x

18.Schwarz L, Lupinacci RM, Svrcek M, et al. Para-aortic lymph node sampling in pancreatic head adenocarcinoma. Br J Surg. 2014 Apr;101:530-538. https://doi.org/10.1002/bjs.94449. Jones AE, Trzeciak S, Kline JA. The sequential organ failure assessment score for predicting outcome in patients with severe sepsis and evidence of hypoperfusion at the time of emergency department presentation. Crit Care Med. 2009;37(5):1649-54. https://doi.org/10.1097/ccm.0b013e31819def97 